\title{
ENTRE PERCEPCIONES Y REALIDADES: LA ADMINISTRACIÓN REAGAN ANTE LA POLÍTICA ECONÓMICA DEL PSOE EN SU ASCENSO AL PODER EN 1982*
}

\author{
JULIO TASCÓN FERNÁNDEZ \\ Universidad de Oviedo \\ juliotf@uniovi.es \\ MISAEL ARTURO LÓPEZ ZAPICO \\ Universidad Complutense de Madrid \\ m.artzapico@gmail.com
}

(Recepción: 26/08/2014; Revisión: 03/11/2014; Aceptación: 30/05/2015; Publicación: 26/11/2015)

1. INTRODUCCIÓN: OBJETIVOS, FUENTES Y MARCO CONCEPTUAL.-2. LAS RELACIONES HISPANO-NORTEAMERICANAS DESDE LA MUERTE DE FRANCO HASTA 1982.-3. EL PROGRAMA ECONÓMICO DEL PSOE EN EL ESPEJO DE LA EMBAJADA NORTEAMERICANA EN MADRID.-4. CONCLUSIONES.-5. REFERENCIAS.-6. BibliOgRAFÍA

\section{RESUMEN}

Tras la muerte del general Franco, los sucesivos gobiernos norteamericanos -tanto republicanos como demócratas- observaron atentamente los cambios socio-políticos que se estaban produciendo en España con objeto de asegurar los intereses estadounidenses en el país. Más allá del componente militar-clave de la relación hispano-norteamericana durante el franquismo- la situación de la economía española aparece como uno de los temas preferentes, tal y como queda al descubierto en los diversos informes remitidos desde la Embajada estadounidense en Madrid durante el proceso de transición

$\left({ }^{*}\right)$ Este estudio se inserta en el proyecto de investigación Historia económica de la relación bilateral entre España y Estados Unidos (segunda mitad del siglo XX y comienzos del XXI), Instituto Franklin-UAH, 2012. Igualmente se ha beneficiado de la financiación derivada de la ayuda de investigación Kenneth Galbraith en temas económicos y políticos relacionados con Estados Unidos. Año 2014. Concedida por el Instituto Franklin-UAH. 
a la democracia. Esta documentación servía a los Departamentos del Tesoro y Estado para evaluar el riesgo país de España en estos complejos años. Apoyándose en estas fuentes primarias, el presente artículo analiza cómo se percibía desde la Administración Reagan la posible llegada al poder de un gobierno socialista y cuál era su balance sobre el programa económico que presentaba el PSOE ante la cita electoral de 1982.

Palabras clave: PSOE; Administración Reagan; economía política; política económica; elecciones 1982

\title{
BETWEEN PERCEPTIONS AND REALITIES: THE REAGAN ADMINISTRATION AND THE ECONOMIC POLICY OF THE SPANISH SOCIALIST PARTY IN ITS RISE TO POWER IN 1982
}

\begin{abstract}
After the death of General Franco, US Governments -Republicans and Democratsgot into a very attentive surveillance on socio-political changes that were taking place in Spain in order to secure US interests in the country. Beyond military issues -one of the main themes considering American-Spanish relationship during the Francoism- the Spanish economic conditions appeared like one preferred topic, as it has been disclosed by different reports sent from Madrid US Embassy during the «Transition to Democracy». These records served to both Treasury and State Departments, in order to assess the country risk of Spain at this time. Based through this kind of primary sources, our paper analyze how Reagan Administration perceived the possible rise to power of the Spanish Socialist Party (PSOE) and which was its own assessment on the economic program presented by the PSOE before 1982 elections.
\end{abstract}

Key words: Spanish Socialist Party (PSOE); Reagan Administration; political economy; economic policy; 1982 elections

\section{INTRODUCCIÓN: OBJETIVOS, FUENTES Y MARCO CONCEPTUAL}

Este artículo profundiza en un tema relativamente poco explorado, el de las percepciones de la Administración Reagan sobre los socialistas españoles y su programa económico si estos lograban llegar a la Moncloa, como acabó sucediendo tras las elecciones de octubre de 1982. Aunque en la última década hayan aparecido trabajos que han aportado enfoques novedosos sobre las relaciones hispano-norteamericanas durante el franquismo y la transición a la democracia -sobre todo en el ámbito de la diplomacia pública-, los aspectos políticos y geoestratégicos de las mismas siguen siendo los más conocidos (1).

(1) A modo de ejemplo: Marquina (1986); Jarque (1998); Viñas (2003); Pardo (2003); TERMis Soto (2005); PowELl (2011) o LeMUS (2011). El creciente interés por los aspectos sobre 
Aunque el estudio de los condicionantes económicos no se haya dejado totalmente de lado, su importancia ha quedado preterida al verse estos reducidos a ser solo otra pieza dentro de un contexto más amplio. Existen diversos análisis acerca de la importancia que tuvo el capital norteamericano en la forja de la economía de la España moderna, al convertirse en el líder destacado desde la década de los sesenta y manteniendo esa preeminencia en el periodo aquí contemplado. Para 1975, dicha importancia se tradujo en la presencia de más de trescientas empresas norteamericanas operando en el territorio y en unas intensas relaciones comerciales que se mantuvieron en los años posteriores, pese al creciente protagonismo de Europa (2).

Como es sabido, la transición española tuvo lugar durante un momento extremadamente complicado en el ámbito económico (3). Cabe recordar que los problemas políticos abordados durante la misma no fueron ajenos a los efectos de las crisis energéticas de los años setenta (4). Sin contar, además, con el hecho de que España estaba sufriendo el agotamiento del modelo de crecimiento desarrollista precisando, por ende, una profunda reforma estructural (5).

A todos estos condicionantes, que pueden ayudar a entender el limitado interés generado hasta la fecha por la dimensión económica de las relaciones entre nuestro país con la potencia americana, hemos de añadir una complicación adicional para el tema específico que nos ocupa. Indefectiblemente, siempre que sale a colación el Partido Socialista Obrero Español (PSOE) junto a los Estados Unidos, el centro de atención se desplaza hacia la posición de esta organización política con respecto al papel que debería jugar España en la política de seguridad occidental (6). La postura abiertamente contraria a la integración española en la OTAN defendida por el partido socialista con anterioridad a su llegada al gobierno fue un marcado elemento distorsionador en la relación entre los representantes estadounidenses y los líderes de la formación política, por más que existieran ciertos matices dentro de este último colectivo (7).

La materia objeto de tratamiento aquí -economía política de las relaciones hispano-norteamericanas- parece pues estar necesitada de una reconsideración que permita arrojar cierta luz sobre ella. Para ello contamos básicamente con documentos recientemente desclasificados por los archivos estadounidenses y que nos permiten introducir nuevos elementos de discusión que pueden conducir

la diplomacia pública y la política cultural de Estados Unidos hacia España ha partido en los últimos años desde la academia española aunque la reciente aparición de la obra de Rosendorf Franco Sells Spain to America podría indicar un giro en esta tendencia. Para un listado de autores y títulos sobre la materia véase LóPEZ ZAPICO (2013): 13-27.

(2) Entre otros estudios podemos mencionar: TASCÓN (2005); PUIG (2005) y ÁlVARO (2011).

(3) FuENTES Quintana (1999).

(4) SUDRIÀ (1987): 340-351.

(5) HERNÁNDEZ (2004).

(6) LiNARES (2008).

(7) RUPÉREZ (1986). 
a construir una visión más global de las relaciones de España con la superpotencia en nuestra historia reciente. La cercanía temporal del periodo contemplado en este artículo (1973-1982) implica la imposibilidad de tener acceso a la mayoría de los documentos que se encuentran custodiados en los archivos públicos de ambos países. Sin embargo, incluso a pesar de este inconveniente, las fuentes norteamericanas son mucho más accesibles que su contraparte española (8). Tal circunstancia ha propiciado que nuestro enfoque se base en la introspección realizada por la Administración Reagan sobre los posibles cambios que, previsiblemente introduciría un gobierno socialista en materia económica si se alzaba con la victoria en las elecciones de 1982. Asunto que para el gigante americano cobraba una especial importancia a tenor del apoyo brindado por las anteriores administraciones a los partidos socialdemócratas en Portugal y Grecia, normalmente por vía interpuesta, como respuesta a la amenaza comunista en ambos países (9).

Nuestra fuente principal son los fondos del Departamento del Tesoro. Estos custodian los informes económicos remitidos regularmente por la Embajada estadounidense en Madrid con objeto de facilitar el trabajo a los analistas de Washington a la hora de evaluar el riesgo país de España. Se debe apuntar que estos mismos informes también eran utilizados por la delegación estadounidense que formaba parte del Comité Económico Conjunto hispano-norteamericano (10). Si bien durante la primera mitad de la década de los setenta la trayectoria del Comité fue un tanto errática, sus funciones quedaron nuevamente reafirmadas en el articulado del Tratado de Amistad y Cooperación firmado por España y Estados Unidos en 1976 (11).

Para complementar la información contenida en estos documentos recurriremos también a otras fuentes. En este sentido, son de ayuda tanto algunas referencias aparecidas en la prensa de la época como los propios programas electorales del Partido Socialista, sin menospreciar diversos testimonios y memorias de los principales protagonistas. Con este bagaje estaremos en condiciones de recomponer de forma bastante precisa la opinión que los observadores de la Administración Reagan tenían acerca del rumbo económico que podría adoptar un gobierno encabezado por Felipe González. Como después comprobaremos, la mayor parte de sus impresiones no tardaron en verificarse y fueron

(8) Molinero (2011) y NiÑo y SANZ (2012).

(9) ChIPMAN (1988) y MuÑOZ SÁNCHEZ (2012)

(10) Esta institución tiene su origen en los esfuerzos del ministro de Asuntos Exteriores, Fernando María Castiella, por ampliar durante los años sesenta los términos de la relación bilateral más allá de las cuestiones militares. Véase LóPEZ ZAPICO (2013): 245-249.

(11) «Acuerdo complementario sobre cooperación económica» contenido en el «Instrumento de Ratificación de España del Tratado de Amistad y Cooperación entre España y los Estados Unidos de América, los siete Acuerdos Complementarios al mismo y ocho Canjes de Notas de 24 de enero de 1976 y del Acuerdo de Desarrollo del Tratado de Amistad y Cooperación, los Anexos de Procedimiento I, II, III, IV, V, VI, VII, VIII, IX-A, IX-B, X, XIII, XIV, XV, XVI y dos Canjes de Notas de 31 de enero de 1976», Boletín Oficial del Estado, n. ${ }^{\circ}$ 267, 6 de noviembre de 1976, 21913. 
correctas, lo que es una buena muestra de la alta calidad de estos informes y, sobre todo, de la habilidad de la diplomacia norteamericana para obtener datos fiables acerca de la realidad política y económica de España. Sin embargo, llama poderosamente la atención cómo esta capacidad de ejecutar con éxito difíciles ejercicios prospectivos no es incompatible con la presencia en los informes de valoraciones que revelan ciertas ideas preconcebidas y juicios de valor, únicamente comprensibles si son entendidos bajo la óptica de las percepciones. En este sentido, las clásicas aportaciones de Robert Jervis sirven para ilustrar con meridiana claridad el notable peso que los factores ideológicos desempeñan en los procesos de toma de decisiones y cómo las percepciones -sean estas correctas o equivocadas- han contribuido a perfilar el comportamiento de los gobiernos hasta la época presente (12).

La economía política tampoco ha sido ajena a la influencia de estos procesos, incluso a pesar de que los actores implicados siempre traten de justificar sus actuaciones escudándose tras sesudos informes y datos contrastados (13). Por ejemplo, la actitud abiertamente antiamericana de importantes sectores próximos al partido socialista contribuyó a generar un difuso sentimiento de pesimismo en el seno de la Administración Reagan sobre el posible entendimiento que, en materia económica, podría alcanzarse con el gobierno resultante de las elecciones de 1982. A pesar de sus esfuerzos para tratar de abstraerse de esta clase de condicionantes, los funcionarios de la Embajada norteamericana en Madrid no siempre tuvieron éxito en su empresa. Esto les llevó a realizar estimaciones que, en muchos casos, destilan un pesimismo mucho más marcado de lo que parecía desprenderse de una coyuntura económica compleja, sin duda, pero no descorazonadora. Se trata, en definitiva, del tradicional modus operan$d i$ de las percepciones en las cuales las representaciones mentales influyen sobre la forma en que aprehendemos la realidad y viceversa (14).

Los ejercicios prospectivos realizados desde la Embajada, posiblemente al operar desde un marco conceptual divergente, siempre se mostraron menos condicionados por el recurso a los estereotipos que aquellos redactados directamente en Washington D.C. Estos consejos de los analistas de la legación norteamericana en España no siempre fueron escuchados ni por los responsables del Departamento de Estado ni por los del Tesoro. Con todo, sí contribuyeron,

(12) Aunque Robert Jervis ha continuado perfeccionando su análisis sobre los procesos de toma de decisión en las sucesivas reediciones del manual que edita conjuntamente con Robert J. Art (2014), sus trabajos acerca del peso de las percepciones aparecidos en la década de los setenta siguen manteniendo su plena vigencia (1971) y (1976). Adicionalmente, son interesantes las aportaciones de VosS y DORSEY (1992), así como las de los autores de una obra colectiva sobre el peso de las percepciones en las relaciones transatlánticas aunque esté centrada en un periodo posterior al contemplado en este artículo FERNÁNDEZ SOLA y SMITH (2009).

(13) Para una introducción al concepto de Economía política internacional véase GiLPIN (1987)

(14) SÁNCHEZ ReCIO (2008). 
indudablemente, a disipar ciertas incógnitas y, sobre todo, a contrarrestar el pensamiento moderadamente pesimista que se respiraba en el seno de la Administración Reagan sobre la capacidad de un gobierno socialista para respetar las reglas del juego establecidas en los ámbitos financieros y comerciales.

Acceder a un material semejante al anteriormente descrito para poder así estudiar los informes manejados por las autoridades españolas resultaría de incuestionable interés pero no parece factible por ahora. Esta accesibilidad nos situaría en disposición de verificar hasta qué punto el antiamericanismo estaba realmente extendido en los estamentos de poder o se trataba, más bien, de un fenómeno que emanaba, en esencia, de las bases de los partidos de izquierda (15). Por el momento, la hipótesis más sugerente es la desarrollada por Carlos Alonso Zaldivar cuando define la relación bilateral como un agregado de desconocimiento por parte americana y antipatía por parte española que generaba un conjunto de lugares comunes o, según sus propios términos, «miradas torcidas» (16). En todo caso, este tipo de sentimientos de incomprensión mutua únicamente son explicables si tenemos en cuenta el contexto político y económico en el cual se insertaron las relaciones hispano-norteamericanas durante la transición a la democracia.

\section{LAS RELACIONES HISPANO-NORTEAMERICANAS DESDE LA MUERTE DE FRANCO HASTA 1982}

Desde la firma en 1953 de los acuerdos bilaterales entre España y Estados Unidos el régimen de Franco había depositado en esta conexión su esperanza de alcanzar, por fin, el perdón por su controvertida relación con las potencias del Eje durante la Segunda Guerra Mundial. El franquismo aspiraba a reincorporarse dentro del orden internacional haciendo gala tanto de la importancia geoestratégica de España en el marco de la Guerra Fría como de su marcado carácter anticomunista. Franco no vaciló en sacrificar importantes parcelas de soberanía, incluyendo la presencia de varias instalaciones militares norteamericanas que disfrutaban de una autonomía casi total, en aras de garantizar su

(15) Pese a que el antiamericanismo español dista mucho de ser un fenómeno únicamente atribuible a la izquierda, como han demostrado las investigaciones de Daniel Fernández (2012), durante la transición y los primeros años de la década de los ochenta parte de los votantes socialistas concentraban su resquemor hacia Estados Unidos en los acuerdos alcanzados durante la dictadura franquista y en la política exterior de la potencia, tachada de imperialista. Véase AzCÁRATE (1988) y SEREGni (2010). El decidido intervencionismo de la Administración Reagan en Centroamérica potenció aún más este tipo de acusaciones, las cuales cobraron especial importancia por el gran protagonismo de Felipe González en América Latina al ejercer como uno de los máximos representantes de la Internacional Socialista en esta zona, BLÁZQUEZ (2006).

(16) Alonso Zaldivar (2003). Resulta significativo que otros analistas, como puede ser el caso de Chislett, asumieran, tan solo hace una década, la necesidad de que entre ambas naciones se produzca una redefinición de sus relaciones (2005). 
propia supervivencia (17). Es conveniente recordar que este acuerdo no incluía una cláusula de defensa mutua, al margen de que las contrapartidas de carácter material siempre fueron consideradas como insuficientes por el lado español. Las sucesivas renovaciones de estos acuerdos en 1963, 1968-1970 y 1975-1976 estuvieron caracterizadas por la necesidad de equilibrar los términos de la relación, alcanzando de una u otra manera alguna clase de compromiso defensivo así como mayores compensaciones, especialmente en lo que concernía al capítulo militar.

Cuando a finales de la década de los sesenta y, de forma aún más palpable, durante la plenitud del tardofranquismo el régimen de Franco comenzó a evidenciar signos manifiestos de agotamiento - no solo en su vertiente político y social sino también en la económica-, el gobierno norteamericano procuró encontrar la fórmula para asegurar sus intereses en España toda vez que el dictador hubiera abandonado la escena. Un claro punto de inflexión fue el derrumbe de la dictadura portuguesa tras la revolución de los claveles pues la crisis en el país vecino tuvo un notable impacto en la concepción del Departamento de Estado hacia la península ibérica y favoreció una necesaria coordinación con las democracias europeas (18).

Las acciones ejecutadas no fueron siempre exitosas y, de alguna manera, su falta de implicación durante algunos de los complejos episodios por los que atravesó el régimen en su fase terminal hizo que Estados Unidos no fuera considerado, por un importante sector de la sociedad española, como un país favorable hacia el proceso democratizador (19). Esto no fue óbice para que, merced al excelente trabajo realizado por sus informantes desplazados al territorio español, los estadounidenses fueran capaces de predecir el carácter relativamente pacífico del cambio que estaba por llegar. Parece innegable el hecho de que en alguno de estos informes puedan ser detectadas ciertas dosis de wishful thinking, rasgo que no minimiza el trabajo desplegado por una diplomacia preparada para hacer frente a casi cualquier eventualidad y que no tardó en identificar a Juan Carlos I como la pieza clave en la nueva relación que habría de establecerse entre los dos países.

La conversión en 1976 de los acuerdos ejecutivos a la fórmula de tratado bilateral tuvo más un valor simbólico que una trascendencia práctica ya que,

(17) VIÑAS (2003)

(18) Sobre el modo en el que la diplomacia norteamericana encaró la crisis abierta en el Portugal post-salazarista, la cual desbordó cualquier previsión realizada desde Washington, véase GOMES y Moreira dE SÁ (2008) o Lemus et al. (2011). Estos trabajos sirven para revelar la existencia de un claro temor de contagio y para explicar el cambio de actitud de Kissinger hacia la península ibérica; así como repasan las actitudes de los embajadores Wells Stabler y Frank Carlucci durante el proceso de transición de ambos países. Esta contextualización puede ampliarse más para entroncar con lo que estaba sucediendo en otras naciones de la cuenca mediterránea y los intereses norteamericanos en el área, GUNTHER et al. (1995).

(19) Powell (2010). 
de algún modo, no dejó de ser una actuación para la galería. Tal circunstancia no debe, sin embargo, hacernos pasar por alto el importante crédito cercano a los mil millones de dólares que fue concedido ese mismo año por parte de un consorcio de bancos norteamericanos. Esta operación parece un claro indicador de la existencia de un alto grado de confianza en el proceso democratizador (20). Es importante destacar que esta confianza no emanó de la posición adoptada por el secretario de Estado Henry A. Kissinger sino que el mérito ha de ser otorgado a la labor desplegada por el equipo de la Embajada norteamericana en Madrid, encabezada desde 1975 y hasta 1978, por el sagaz diplomático Wells Stabler (21). Kissinger mostró evidentes síntomas de miopía política hacia España al limitar su actuación a sugerir fórmulas poco o nada imaginativas basadas, preferentemente, en la imposición de un ritmo lento en el proceso de cambio y en su negativa a tender puentes hacia los miembros de la oposición democrática, supeditando esta tarea a la buena voluntad de los líderes europeos (22). Afortunadamente para los intereses norteamericanos, el embajador Stabler -respaldado por su subalterno Samuel D. Eaton- era de la opinión de que para garantizar una relación bilateral en el largo plazo sería oportuno reunirse con los principales líderes de la oposición democrática (dejando al margen al partido comunista por razones obvias), dado que, en el futuro, estos podrían llegar a alcanzar responsabilidades políticas en el gobierno español (23).

Los encuentros comenzaron a tener lugar antes del fallecimiento del general Franco y, aunque nunca fueron considerados una prioridad, se mantuvieron a lo largo de los años. Para poder evaluar el significado real de los mismos contamos con la entrevista que Stabler mantuvo con Felipe González en octubre de 1975 en la cual el secretario general del PSOE confirmaba que los socialistas estaban dispuestos a ofrecer a Juan Carlos un cierto margen de maniobra, siempre que el futuro monarca demostrara con sus acciones un verdadero compromiso con los ideales democráticos (24). La información obtenida en esta clase de contactos -primero informales y más tarde abiertamente reconocidos toda vez que las distintas formaciones políticas fueron legalizadas-ayudó a los consejeros de la

(20) Powell (2011): 375.

(21) POWELl (2007).

(22) ORTUÑo (2005) y Powell (2011). El factor alemán resultará decisivo en estas lides y no es posible entender la forja del liderazgo moderado y pragmático de Felipe González sin mencionar la poderosa influencia de Billy Brandt y la ayuda que el PSOE recibió de su homólogo germano, MUÑOZ (2012).

(23) EATON (1981) y StABLER (1987).

(24) National Archives and Records Administration (NARA) RG 59, Records of Henry A. Kissinger 1973-1977, box 24, Telegrama de la Embajada norteamericana en Madrid al Departamento de Estado (7481), 26 de octubre de 1975. Luis Yáñez, que ostentó el cargo de secretario de relaciones internacionales del PSOE entre 1975 y 1979, ha comentado que durante aquel periodo: «tuve varias entrevistas, algunas solo y otras junto a Felipe González, con el embajador de los Estados Unidos en España y con algunos de sus consejeros», PAPELL (1991): 113. 
Embajada norteamericana a la hora de confeccionar informes lo más precisos posibles con los que hacer partícipes a sus superiores acerca del proceso de transición a la democracia. Un buen ejemplo de su labor es observable cuando anticiparon con cierta antelación los pobres resultados que iba a obtener el Partido Comunista Español (PCE) en las elecciones del 15 de junio de 1977, a pesar de haber sido el grupo opositor mejor organizado durante la dictadura franquista.

La llegada a la Casa Blanca en 1977 de una nueva Administración tras la victoria en las urnas del candidato demócrata Jimmy Carter no implicó cambios sustanciales en los términos de la relación hispano-norteamericana, manteniéndose la posible integración de España en la OTAN como el asunto más destacado (25). La posición de los diferentes gobiernos encabezados por Adolfo Suárez se mantuvo en una incómoda ambigüedad a este respecto, revelando la existencia de fuertes divisiones internas sobre la materia. Esta falta de acuerdo fue aprovechada por el partido socialista para convertir su rechazo a la adhesión de España a la OTAN en una de las ideas-fuerza de su discurso político. En esa posición anti-OTAN defendida desde las filas del PSOE existía, al menos en las altas esferas del partido, un importante componente de oportunismo político que condujo a alguno de sus miembros a cometer ciertos excesos verbales de los que más tarde habría de arrepentirse (26). De hecho, si analizamos en profundidad los programas electorales de esta formación durante el periodo estudiado, es posible encontrar en ellos ciertas ambigüiedades -el fuerte sentimiento europeísta esgrimido por los expertos socialistas en política exterior no era incompatible con la OTAN- que años después serán utilizadas durante la agria controversia que precedió al referéndum de 1986 (27). Los miembros del Comité Federal del PSOE eran plenamente conscientes de su incapacidad para ser considerados como una alternativa real a la UCD fuera de nuestras fronteras si no eran capaces de mostrar una postura menos dogmática. Este hecho ayudaría a explicar los motivos que llevaron tanto a Felipe González como a Luis Solana - por aquel entonces al cargo de la política de defensa del partido socialista- a visitar los Estados Unidos entre 1977 y 1978 con la intención de transmitir un mensaje más moderado (28). Estos gestos no fueron siempre correctamente

(25) Véase por ejemplo SÁNCHEZ-GIJÓN (1978) y RodRIGo (1995).

(26) La polémica existente sobre si este rechazo a la OTAN era genuino o una mera estrategia, derivada de un cálculo electoral interesado que reflejaba la obsesión del socialismo español por alcanzar la presidencia de gobierno, ha generado un intenso debate. Este fue pronto recogido por la historiografía, como queda de manifiesto en los ensayos compilados por GIL y TULCHIN (1989), manteniéndose vigente con posterioridad, ANDRADE BLANCO (2007).

(27) «Elecciones 77. Programa del PSOE», Programa electoral del PSOE, 1977; «Elecciones 79. Programa del PSOE», Programa electoral del PSOE, 1979; y «Por el cambio. Programa Electoral del Partido Socialista Obrero Español», Programa electoral del PSOE, 1982.

(28) «Felipe González, a Estados Unidos», El País, 15 de noviembre de 1977; y Carta de Luis Solana al senador estadounidense Clairborne Pell (fechada el 17 de abril de 1978) en la cual Solana agradece a Pell su amable acogida durante su visita a los Estados Unidos junto al también 
interpretados desde las instancias norteamericanas pero, al menos, contribuyeron a mantener abierta la vía para un futuro entendimiento (29).

Sin embargo, el escenario pareció complicarse seriamente para los socialistas españoles tras producirse la victoria electoral del republicano Ronald Reagan en los comicios de noviembre de 1980. El nuevo presidente se apresuró a marcar las diferencias con su predecesor, introduciendo cambios sustanciales en la política exterior estadounidense con el fin de reivindicar la fortaleza de la superpotencia en la arena internacional (30). Esta nueva actitud no tardó en dejar su impronta alrededor del globo y, para el caso español, el episodio que ha perdurado en el tiempo fue la torpe actuación del secretario de Estado Alexander Haig durante el intento de golpe de Estado del 23-F que dio pábulo a todo tipo de suspicacias e hipótesis sensacionalistas no siempre ajustadas a la realidad (31).

En este complicado ambiente, la decisión del presidente Calvo Sotelo de acelerar los trámites para facilitar la entrada de España en la OTAN -la cual quedó formalizada el 30 de mayo de 1982- otorgó a los socialistas renovados argumentos con los que atacar al gobierno centrista. De hecho, algunos miembros del PSOE aprovecharon la tormenta política desatada durante los convulsos meses que sucedieron a la dimisión de Adolfo Suárez para renovar ciertos dejes antinorteamericanos. Incluso Felipe González criticó descarnadamente la controvertida inhibición de la Administración Reagan durante las delicadas e interminables horas que duró la retención de los diputados en el Palacio de Congresos el 23-F (32). Estas severas declaraciones se produjeron en el marco de una visita de González al Reino Unido, y la Embajada norteamericana en Londres expresó su preocupación dado que el secretario socialista repitió sus duras palabras en los encuentros privados que mantuvo con lord Carrington, a la sazón ministro de Asuntos Exteriores británico, y con el político socialista David Owen (33).

socialista Enrique Múgica. Special Collections University of Rhode Island, Claiborne Pell Papers, box 37.

(29) Quizás el guiño más recordado por su expresividad sea la afirmación realizada por Felipe González en el marco de una reunión de la Internacional Socialista celebrada en Venezuela en 1978: «prefiero morir de una puñalada en el metro de Nueva York que vivir «tranquilo» treinta años en Moscú». $A B C, 12$ de noviembre de 1978, p. 77. Por su espectacularidad esta frase ha sido reinventada hasta la saciedad incluyendo todo tipo de variantes.

(30) Melanson (2005): 128-190. Para una contextualización general sobre las diferencias más notables entre las políticas implementadas por Ronald Reagan frente a sus antecesores y el impacto de la revolución conservadora, véase WILENTZ (2009). Sobre la política exterior del presidente republicano y las inconsistencias entre sus formulaciones teóricas y su puesta en práctica, BELL (1989).

(31) LÓPEZ ZAPICO (2011).

(32) «Comment by Haig Draws Fire in Spain», New York Times, 12 de marzo de 1981.

(33) Telegrama de Streator al Departamento de Estado, 18 de marzo de 1981 (05162) accesible en foia.state.gov/searchapp/DOCUMENTS ffoiadocs\622f.PDF.. Estas aseveraciones fueron posteriormente matizadas por el político sevillano, según se recoge en uno de los telegramas 
La problemática de la integración de España en la OTAN se solapó con la negociación de la renovación del tratado bilateral, vía que Washington consideraba ya agotada (34). Por ello, cuando todos los indicadores eran favorables para la victoria del PSOE en las elecciones de 1982, resulta fácilmente comprensible la preocupación existente entre los miembros del Departamento de Estado acerca del comportamiento de un futuro gabinete socialista que estaría al cargo de gestionar los acuerdos alcanzados con el gobierno de la UCD. Obviamente, la principal inquietud radicaba en el rumbo que iban a adoptar los socialistas en materia de seguridad y defensa (35). Sin embargo, los responsables de la legación estadounidense en Madrid concedían la misma importancia al programa económico que sería implementado por el PSOE, sabedores de que un cambio radical en este campo podría cortocircuitar las relaciones hispanonorteamericanas. Esto explica la conveniencia de revisar los informes generados por los consejeros económicos de la Embajada durante 1982 con objeto de comprender mejor sus percepciones así como la hoja de ruta que, bajo su criterio, debería seguir la Administración Reagan en su trato con el nuevo gobierno socialista.

\section{EL PROGRAMA ECONÓMICO DEL PSOE EN EL ESPEJO DE LA EMBAJADA NORTEAMERICANA EN MADRID}

La década de los ochenta casi se había inaugurado con la sacudida que, para la ya debilitada economía española, supuso el segundo shock del petróleo. El brusco encarecimiento del precio internacional del crudo no solo enturbió las perspectivas de una pronta recuperación de la economía global sino que, para el caso concreto de España, supuso la evaporación de los efectos positivos que habían cosechado las medidas promovidas por los Pactos de la Moncloa (36). La crisis de la industria, el creciente desempleo, la elevada inflación y el preocupante déficit público se alzaban como los principales retos a los que el gabinete de Calvo Sotelo debía hacer frente. Un informe de la CIA, fechado en febrero de 1982, sostenía que los problemas presupuestarios podrían acabar dando el golpe de gracia al gobierno. Una caída que, según los analistas norteamericanos, beneficiaría a un partido socialista destinado a jugar un notable papel en las siguientes elecciones (37).

que Barbour envió al Departamento de Estado el 18 de marzo de 1981 (03508), en foia.state.gov/ searchapp/DOCUMENTS/5-FY2014/F-2011-25964/DOC_0C05225018/C05225018.pdf.

(34) PORTERO (2003).

(35) POWELl (2011): 579-594.

(36) Trullén (1993); García Delgado y Jiménez (2003); Estefanía (2007): 239-256 y CABRERA (2011).

(37) «Monthly Warning Assessment: Western Europe», Memorando para el Director y Subdirector de la CIA fechado el 19 de febrero de 1982. 
Por tanto, la inestabilidad política de este periodo, sumada a la descomposición interna que estaba sufriendo el partido en el gobierno, no parecía ser el mejor escenario para adoptar las medidas necesarias que permitieran enderezar el rumbo de la economía española (38). Sin embargo, existen luces y sombras acerca de los objetivos conseguidos con las políticas económicas emprendidas. Suele darse una valoración positiva a la política económica de la UCD amparada, al menos, en que la herencia recibida era cuando menos muy compleja (39). Este nuevo shock energético se afrontaría recurriendo a las herramientas político-económicas disponibles por aquel entonces: Pactos de la Moncloa, reforma fiscal y diversas medidas de ajuste (40). Además, para atajar los desajustes existentes en los costes de trabajo se creó la cultura de la «inflación esperada». A su vez, se desvió la presión igualitaria del instrumento salarial al fiscal, al tiempo que se introducía un sistema impositivo y de gasto público en línea con Europa. Se redujo la inflación a la mitad y cuando la UCD dejó el gobierno esta se había contenido hasta el 13,2 por ciento (41). El debate sobre la empresa pública que se había originado en los años sesenta presagiaba y hacía prever la era de las privatizaciones que acaecería durante el siguiente ciclo político económico, ya con los socialistas al timón (42). Resulta sintomático que el propio Miguel Boyer, quien ocupó la cartera de Economía, Hacienda y Comercio durante el primer gobierno socialista, sostuviera en 1992 que:

[La política por él conducida al frente del Ministerio] fue una continuidad de la de Leopoldo Calvo Sotelo; lo que pasa es que a él le pilló lo peor del ciclo y muy debilitado políticamente. Nosotros también teníamos el ciclo bajo, pero disponíamos de una gran fuerza política, y un líder indiscutible capaz de aunar sectores muy diversos y con un excelente instinto para los problemas económicos (43).

(38) Hopkin (2000).

(39) La inflación galopante rozaba el $30 \%$ y su tendencia era creciente. A la par, el paro avanzaba de forma diferente al resto de Europa. En un amplio informe de la CIA sobre el grave problema del desempleo juvenil en los países industrializados, España aparecía como la nación más castigada con unas cifras que, para 1981, rozaban el 35\% de la población que estaba en el segmento de edad que iba entre los 15 y 24 años. Solo Portugal se acercaba al caso español rondando el 24\%; mientras que el resto de potencias occidentales oscilaban entre el 5 y el $20 \%$ como máximo. «Industrial Countries: The Youth Unemployment Problem. An Intelligence Assessment», Directorate of Intelligence, julio de 1982.

(40) FuENTES Quintana (1990).

(41) Haciendo una enumeración sumaria de las principales intenciones así como de ciertos logros se puede afirmar que: se profundizó en la liberalización del sistema financiero; se reguló el mercado de trabajo dentro del contexto político de la época; se fortaleció la política monetaria y se evitó una potencial crisis financiera; se creó un contexto de «consenso» en amplias partes de la política económica que facilitó una «Constitución por consenso», etc. GÁMIR (2008): 36-37.

(42) Segura (1998); Bel y Costas (2001); Comín y Díaz Fuentes (2004): 253.

(43) Maluquer De Motes (2008): 256. Aunque con un tono mucho más ácido por su fuerte implicación en el asunto, también Leopoldo Calvo Sotelo era de la opinión de que «las líneas generales de la política económica socialista en sus primeros años son las mismas que orientaron la política económica de UCD, aplicadas -eso sí- con la mayor eficacia que da la mayoría absoluta. Es curioso que fuesen los socialistas, entonces en la oposición, los que impidieran 
Los analistas económicos de la Embajada norteamericana en Madrid observaron, con extremo cuidado, todas y cada una de las medidas adoptadas por el gabinete de Calvo Sotelo con el fin de mantener a sus superiores en Washington correctamente informados sobre la situación política y económica de España. Resulta notable comprobar que las conclusiones recogidas en uno de sus múltiples informes, enviado concretamente en junio de 1982, corroboran las opiniones expresadas por Boyer justo una década después:

In summary, the Spanish economy is in transition. It is undergoing a structural adjustment process which, over the medium term, should strengthen its ability to produce and compete in world markets. Unfortunately this process also brings with it considerable social costs, primarily manifest in joblessness. Structural adjustment to new conditions is not easy, especially for the political leaders who must make decisions which may adversely affect one sector of society or another. It is to its credit that Spain has already begun to make these decisions. Although more difficult choices still remain, Spain's forthright tackling of its past problems indicates that intellectual and material resources of the country are up to the tasks it faces in the future (44).

Esta confianza en la capacidad española para hacer frente a la complicada situación económica parece indicarnos que tanto el controvertido embajador Terence Todman como su equipo eran bastante optimistas acerca del futuro inmediato del país (45). Sin embargo, esta percepción se veía muy sesgada cuando se trataba de analizar el programa económico que sería aplicado por el PSOE en caso de que obtuviera la victoria en las elecciones de 1982. Por ejemplo, una de las circunstancias que más inquietaba a la diplomacia norteamericana era la posibilidad de que, una vez en el poder, los socialistas pusieran en marcha una política nacionalizadora (46). En realidad, esta incógnita flotaba

muchas veces a UCD llevar adelante una política económica ortodoxa, que sin embargo luego harían ellos...» [cursivas en el original], CALVo Sotelo (1990): 161.

(44) Para evitar posibles interpretaciones erróneas, hemos optado por respetar el idioma original de los textos recogidos en el presente artículo. NARA, RG 56, General Records of the Department of the Treasury (GRDT), Office of the Assistant Secretary for International Affairs (OASIA), Records Relating to Portugal, Italy and Spain 1976-1981 (RRPIS), box 2: Economic Portion of Spain's Economic Trends Reports, 24 de junio de 1982.

(45) El nombramiento de Todman para cubrir el puesto que quedó vacante tras la marcha de Stabler en mayo de 1978 se produjo no sin cierta polémica. La prensa española recibió al nuevo embajador con gran suspicacia, generando un clima adverso que se mantuvo durante el resto de su misión, resultando sorprendente su continuidad tras la derrota electoral de Carter. Incluso se llegó a ligar el destino del diplomático con la intentona golpista del 23-F sin que existan testimonios sólidos que verifiquen esta hipótesis, LÓPEZ ZAPICO (2011). Todman, que siempre fue visto con recelo por buena parte de la cúpula socialista, acabó siendo relevado en 1983 por Thomas Enders quien mantuvo unas turbulentas relaciones con el ministro de Exteriores FERNANDO MORÁN, POWELL (2011): 595-604.

(46) La cuestión de las nacionalizaciones y el futuro de la industria pública apareció de forma recurrente en las publicaciones del partido durante los meses previos a los comicios de 1982. MARÍN (2008): 45. 
en el ambiente de una manera interesada ya que algunos altos cargos socialistas gustaban de mantener al respecto cierta ambigüedad que sirviera para dar falsas expectativas a sectores concretos de la sociedad. A la vista de su actuación posterior, todo parece indicar que bien podría haberse tratado de una estrategia perfectamente calculada para atraer votos de las clases trabajadoras y más desfavorecidas (47). Los tiempos habían cambiado aunque no tanto el discurso. Esto ayudaría a explicar por qué la formación socialista que en 1976 propugnaba la necesidad de acometer nacionalizaciones fuera la misma que, en la práctica, comenzara en España el proceso privatizador durante la década de los ochenta (48). El PSOE se presentó a los comicios de 1982 bajo la premisa del cambio, si bien el contenido real que se escondía tras este impactante eslogan electoral no fue plenamente revelado, al menos en su faceta económica, hasta su victoria en las urnas (49). De hecho, cuando el equipo económico del PSOE se vio en la tesitura de tener que desarrollar su programa de gobierno el controvertido tema de las nacionalizaciones aparecía todavía como algo pendiente que habría de ser perfilado:

Carlos Solchaga, en unas recientes declaraciones a La Gaceta del Norte, puntualizó que «si alguien piensa que nosotros vamos a nacionalizar para mantener puestos de trabajo, o para crear otros nuevos, esto constituye una deformación de la realidad». Para añadir, a renglón seguido, que si se produce una situación de abandono empresarial como respuesta a la constitución de un gobierno socialista, «no nos habrá de temblar el pulso en el momento de utilizar cualquier procedimiento, incluidas las nacionalizaciones» (50).

La inteligencia norteamericana incluso fue capaz de detectar la existencia de un contencioso interno sobre esta materia que tendría como protagonistas a Felipe González y Alfonso Guerra. Resulta sintomático que el futuro presidente de gobierno comenzara a aparecer ante los ojos estadounidenses como un político moderado, mientras que Guerra era vinculado a tendencias más izquierdistas:

Alfonso Guerra, the Socialists' second in command, is challenging assurance by Secretary General Felipe Gonzalez [sic] that the party will not undertake na-

(47) Durante el XXVII Congreso del PSOE se aprobó un programa de corte radical cuyo ideario parece hoy día extraño. Suponía el mantenimiento de la propiedad privada solo para las pequeñas empresas en las que se entremezclaba o confundía la propiedad con la gestión de su actividad. Se apostaba desde postulados marxistas, dentro de una economía planificada, por un programa de nacionalizaciones que afectaría a las grandes empresas industriales y de servicios: banca, eléctricas... así como a las agrarias. En 1979 el partido socialista renunció oficialmente al marxismo en un Congreso extraordinario antecedido por una extensa polémica, si bien todavía a comienzos de los ochenta eran frecuentes algunas manifestaciones un tanto extremas en materia económica. Véase AvILÉs (2013).

(48) Sánchez Domínguez y Ortega Almón (2001).

(49) BERMEO Y GARCÍA-DURÁN (1994).

(50) «El PSOE prepara un programa económico de gobierno», El País, 6 de enero de 1982. 
tionalizations if it wins the next election. [...] Gonzalez [sic] believes he would need the acquiescence of business leaders to govern, however, and has been campaigning hard to win their trust. The quarrel within the leadership reflects a longstanding division between Gonzalez [sic] and the more leftist rank and file. Guerra's challenge, however, is probably less a show of strength than an attempt to arrest the decline on the left. Guerra fears that a continued strong showing in opinion polls will further strengthen Gonzalez's [sic] moderate inner circle, which would be the heart of a new cabinet. He is convinced that the Socialists cannot afford to discourage leftists in the party organization from working for victory at the polls. The deputy secretary general appears to be trying to influence Socialists' eventual campaign and possible government by pulling Gonzalez [sic] back to the left. For the moment, however, he probably has cost the party votes in the crucial center of the electorate, particularly in the business community, and he may have increased the misgivings of military and civilian rightists about the party's ability to govern (51).

En el verano de 1982 la victoria socialista parecía más que inminente. Tal circunstancia obligó a la Embajada norteamericana a redoblar sus esfuerzos para conocer en detalle los planes económicos de quienes estaban destinados a remplazar al gabinete de Calvo Sotelo. Fruto de este interés será un completo informe que fue remitido a los Departamentos del Tesoro y de Estado así como a las principales legaciones estadounidenses en Europa. Por su notable interés extractaremos a continuación los párrafos más significativos de este documentado dossier:

Talks with a number of economists within the Spanish Socialist Workers Party (PSOE) indicate that if they could design the economic program of a socialist-led government, it would be pragmatic, realistic and designed to get the Spanish economy moving out of the economic stagnation which has characterized the past several years. To bring about this economic reactivation, PSOE economists want to ask for more sacrifices from labor that were asked by the current Government of the Union of the Democratic Center Party (UCD), but some PSOE politicians and the Socialist dominated General Union of Workers UGT) could thwart their plans. The Socialists economists also want more incentives to business than the UCD in an effort to stimulate job-creating investment. Although they speak wistfully about ridding Spanish society of the vestiges of the Franco Regime, they plan to use these Francoist economic tools to the utmost in carrying out their policies. The economists of the PSOE realistically do not expect a marked improvement in the Spanish economy in the short-run, and this is a cause for worry. They fear that the lack of instant results would sour the public on the solutions of the left and provoke a swing to the right in an effort to go back to the «better life under Franco» which remains in the public memory. The economic theorists of the party may well find that the

(51) National Intelligence Daily (Cable), 3 de marzo de 1982. Aunque en el informe no se mencione la composición de este círculo moderado en torno a González podemos intuir que hace referencia a quienes serían su equipo de confianza ya en la Moncloa, Feo (1993). Sobre la existencia de un pluralismo interno dentro del PSOE que acabó generando diferentes luchas internas véase MATEOS (2013). 
political imperatives of day-to-day government or party ideologues will modify their economic outline to such an extent that the program could become more political and less pragmatic (52).

Como puede apreciarse en los objetivos arriba enunciados, la receta propugnada por los socialistas en materia económica poco o nada tenía que ver con lo que muchos de sus millones de votantes esperaban. En otras palabras, el espejo americano reflejaba una imagen más cercana al proyecto a la postre implementado que la dibujada en su cabeza por una parte del electorado que confiaba, tal vez ingenuamente, en que la promesa de cambio se tradujera en una política económica de corte izquierdista (53).

Tras la introducción, los analistas norteamericanos descendían al detalle para revisar una por una las claves del proyecto económico del PSOE:

Typical PSOE economic policies would be based on increased Government control of the credit system, expansionary fiscal policy, increased taxation to moderate the inevitable fiscal deficit, continued reduction in real wages, restored business profits and investment, more foreign investment and rapid depreciation of the exchange rate. The goal of these policies would be to stimulate economic growth and halt both the loss of jobs and the increase in unemployment (54).

Otra medida diseccionada por los diplomáticos estadounidenses era la política monetaria, cuyo objetivo se fijaba en reducir las tasas de interés a los pequeños y medianos empresarios para favorecer la creación de empleo algo que, según sostenía el informe, no implicaba ni la nacionalización del sistema bancario ni la reducción del gasto del sector público (55). Precisamente, acerca del gasto público, los redactores del documento comentaban que este no iba a poder superar al efectuado por la UCD. De hecho, los socialistas pronto se verían impelidos a subir los impuestos para poder hacer frente al déficit a corto plazo. Esta nueva política fiscal tenía todas las trazas de acabar recayendo sobre los consumidores pues los analistas dudaban de la capacidad del nuevo gobierno a la hora de poder reducir el fraude y la evasión de impuestos. Por todo ello, este incremento se perfilaba como una carga que iban a tener que soportar, sobre todo, aquellos con menores ingresos; sector de donde procedía buena parte del

(52) NARA, RG 56, GRDT, OASIA, RRPIS 1976-1981, box 2: The Outlines of a Socialist Economic Policy in Spain, 14 de julio de 1982.

(53) ESTEFANÍA (2013).

(54) NARA, RG 56, GRDT, OASIA, RRPIS 1976-1981, box 2: The Outlines of a Socialist Economic Policy in Spain, 14 de julio de 1982.

(55) NARA, RG 56, GRDT, OASIA, RRPIS 1976-1981, box 2: The Outlines of a Socialist Economic Policy in Spain, 14 de julio de 1982. Es sintomático que, una vez alcanzado el poder, la cúpula socialista procurara reunirse con los principales representantes del mundo empresarial y de la banca. El objetivo no solo era calmar sus recelos sobre la futura política que sería adoptada en materia económica sino contar con su conformidad con las medidas que se contemplaban para combatir la crisis existente, CABRERA y DEL REY (2011): 366-367. 
apoyo electoral del PSOE (56). Se trataba solo de otra de las varias contradicciones detectadas por los consejeros de la Embajada norteamericana en el proyecto económico del partido socialista. Asimismo, consideraban que la política salarial iba a ser uno de los aspectos más complicados para el gobierno del PSOE porque:

The Party's economists believe that it is necessary to have a 2 to 3 percent reduction in real wages in order to reduce labor costs, moderate inflationary pressure and hold down personnel costs in the Government budget (57).

Este enjundioso informe también hace hincapié en lo referente a los factores económicos externos, subrayando que, pese a la imposibilidad del PSOE para controlarlos, iban a influir notablemente en la agenda económica del nuevo gobierno. Donde los socialistas sí que podrían jugar un papel más relevante sería en sus intentos por atraer a las inversiones extranjeras dado que eran conscientes de que el país habría de depender tanto de tecnología de importación como del know-how externo en materia de dirección para lograr mejorar la eficiencia y competitividad de la industria española. Igualmente, se hace mención a un aspecto que por aquel entonces había levantado cierta controversia como era la posibilidad de que los socialistas optaran por emular el modelo económico francés. Para los norteamericanos no parecía existir duda de que los líderes del PSOE habían descartado dicho modelo por su falta de efectividad:

Spanish Socialists are quick to distance themselves from the economic policies of the Mitterrand Government. These policies (nationalization, reduced work hours to spur employment, etc.) strike the Spanish as being old-fashioned and nor responsive to current world economic circumstances. The PSOE seeks to win over the Spanish businessman by providing incentives to spur job-creating investment. The moderation the Party espouses is not a facade, therefore, but rather a political necessity, given the possibilities for political and social turbulence in Spanish society (58).

(56) NARA, RG 56, GRDT, OASIA, RRPIS 1976-1981, box 2: The Outlines of a Socialist Economic Policy in Spain, 14 de julio de 1982.

(57) NARA, RG 56, GRDT, OASIA, RRPIS 1976-1981, box 2: The Outlines of a Socialist Economic Policy in Spain, 14 de julio de 1982. En el informe se detecta acertadamente que esta cuestión iba a convertirse en un claro punto de fricción con las pretensiones de los sindicatos. Especialmente con una UGT que iba a competir en las elecciones sindicales casi al mismo tiempo que los comicios generales. Las políticas de empleo del primer gabinete socialista -insertas dentro del denominado proceso de reconversión industrial- sirvieron para que el PSOE fuera acusado de sumarse a las recetas neoliberales de estímulo de la oferta que caracterizaron a los gobiernos de Margaret Thatcher y Ronald Reagan, GÁLVEz (2013). Otros autores sostienen, por el contrario, que el modelo socialista se apartaba de las Reaganomics al seguir otorgando a los poderes públicos un activo papel en la resolución de los problemas económicos a través de un gasto canalizado, preferentemente, hacia educación e infraestructuras, GUNTHER, MONTERO y BOTELLA (2004): 355-363. Sobre el significado del término Reaganomics y su traducción práctica desde una óptica no excesivamente crítica, véase NISKANEN (1988).

(58) NARA, RG 56, GRDT, OASIA, RRPIS 1976-1981, box 2: The Outlines of a Socialist Economic Policy in Spain, 14 de julio de 1982. 
La previsión iba en la dirección correcta. El propio Felipe González, apenas un par de meses después de ocupar la Moncloa, reconocería ante el secretario de Estado norteamericano George P. Shultz la «deuda moral» contraída con su homólogo francés François Mitterrand:

«President Mitterrand», Gonzalez [sic] replied, «came in with a big majority on a Socialist ticket, just as I did. He put the Socialist program into place, and the result was a catastrophe for France. Therefore, I have learned something: don't implement the Socialist program. Use the marketplace. Encourage investors. That is what I am going to do». And that is what he did, with great success (59).

Cabe destacar que fue el ya mencionado Miguel Boyer quien jugó un papel decisivo en la determinación de desechar el modelo económico francés, tal y como años más tarde manifestó:

Yo mismo había advertido a Felipe González en 1981, que convenía moderar todo entusiasmo respecto a los socialistas franceses -que acababan de ganar las elecciones- así como distanciarse de sus recetas, pues el fracaso de su política económica iba a ser de manual y sonado, y podría servir, en la política española, de argumento contra nosotros (60).

Por último, para cerrar su informe, los observadores estadounidenses llegaban a la siguiente conclusión:

If the PSOE can get by the short-run, it has grandiose plans to restructure the Public Administration system by making it more responsive to the needs of local Governments, reforming the education system to make it better able to meet the needs of modern society, and creating conditions to improve social justice and reduce class friction. These long-run goals can only be reached by dealing effectively with Spain's pressing economic problems, and the Socialists are ready, in theory, to administer the tough medicine needed to improve the economy. It remains to be seen, however, whether the political realities of day-to-day government will allow these economic remedies to be applied (61).

Además de la información proporcionada desde la Embajada, la Administración Reagan contó igualmente con los datos que emitían los servicios de inteligencia norteamericanos, donde, como ya hemos visto en este artículo, se introducían también valoraciones sobre economía (62). Así, en un documento

(59) SHULTZ (1993): 150.

(60) EsTEFANÍA (2007): 243.

(61) NARA, RG 56, GRDT, OASIA, RRPIS 1976-1981, box 2: The Outlines of a Socialist Economic Policy in Spain, 14 de julio de 1982.

(62) Con todo, la información recibida por diversas fuentes, en lugar de resultar de ayuda y contribuir a enriquecer el conocimiento de la situación española, a veces propiciaba cierta confusión. Es más, esta podía incluso llegar a un más alto nivel de enmarañamiento toda vez que intervenían los diversos asesores presidenciales. Un claro ejemplo de ello es el episodio que relata Todman a la llegada del secretario de Estado Shultz para entrevistarse con Felipe González poco después de su investidura. El embajador se dio cuenta de que las notas que él había redactado para preparar la visita habían sido alteradas por los funcionarios de Washington. Frontline Diplomacy. 
dedicado a analizar el futuro político de Alianza Popular, estos afirmaban que los dictados en materia económica de los conservadores -como cabeza virtual de la oposición- estarían muy condicionados por la política que implementasen los socialistas:

At least initially, the socialists would probably tailor their economic policies accordingly in an effort to take the edge off of business aversion to them. Fraga will continue to speak out strongly on business issues, but development of an accommodation between the business community and a leftist government would remove much of the force from his arguments (63).

\section{CONCLUSIONES}

Por todo lo expuesto, queda claro que la Administración Reagan encaró con relativo optimismo la posibilidad de alcanzar un entendimiento con el gobierno socialista salido de las urnas en 1982. Una de las claves radicaba en la continuidad del rey Juan Carlos I al frente de la jefatura del Estado, figura de especial interés para Washington por su trayectoria durante el proceso de transición (64). Además, el pragmatismo que se presuponía a Felipe González, que explica la cálida acogida que tuvo la victoria del PSOE en la prensa norteamericana, no tardó en ser corroborado por los hechos (65). El principal escepticismo partía de los apartados del discurso político del PSOE relacionados con la estrategia occidental de seguridad y defensa, uno de los nudos gordianos de la relación hispano-norteamericana (66). Pero más allá de estas reticencias, el balance parecía esperanzador:

Manuscript Division, Library of Congress, Washington D.C. Entrevista con Terence A. Todman, 13 de junio de 1995.

(63) «The Spanish Popular Alliance: A New Contender for Power. An Intelligence Assessment», Directorate of Intelligence, octubre de 1982.

(64) Esta sintonía queda patente en una anotación realizada por Reagan en sus diarios con motivo de la visita de los reyes de España a Washington en octubre de 1981: «Our meeting were fruitful even though he is very conscious that under the Constitutional Monarchy he can not declare policy. We've established a good raport [sic]» REAGAN (2009): 74. Cabe recordar que en esas mismas fechas los servicios de inteligencia soviéticos llegaron a hacer circular una supuesta misiva de Reagan a Juan Carlos en la que el mandatario norteamericano presionaba para que España se adhiriera a la OTAN, ANDREW (1995): 465 [la supuesta carta aparece reproducida en las páginas centrales de la obra].

(65) «Liberation in Spain», The New York Times, 31 de octubre de 1982, p. E18. En el viaje de González a Washington, en junio de 1983, Reagan lo describe como «He's sharp, a bright, personable, young, moderate \& pragmatic socialist», REAGAN (2009): 239. Más allá de las referencias a la visita presidencial de Reagan a Madrid en 1985 y algunas menciones a la postura socialista respecto a América Latina, las referencias a España en estos diarios son muy reducidas en consonancia con la autobiografía del presidente donde estas son extremadamente tangenciales o en sus notas donde el país ni siquiera aparece citado, REAGAN (1990) y (2011), respectivamente.

(66) PARDO (2011). Según sostiene Antonio Marquina Barrio, en una información que no hemos podido contrastar por otra vía, Felipe González en agosto de 1982 habría revelado ante los 
We believe that the election outcome has enhanced the prospects for long-term political stability. [...]. In our view, the longevity of the new party structure will depend largely on the political maturity of the victorious parties. The Socialist faces the greatest challenge, having to satisfy both their new centrist supporters and their traditional leftists constituency. If the party's program fails to arrest the decline in Spain's economy, radicals may push for measures that would antagonize rightists power centers and the military. Nevertheless, we believe that a Socialists' disciplining and pragmatism will enable them to steer a moderate course in the new future (67).

En el ámbito económico las visiones prospectivas realizadas por la diplomacia norteamericana resultaron ciertamente ajustadas. Incluso a pesar de las precauciones que, por ejemplo, quedaban patentes en ese documento fechado en julio de 1982 que hemos analizado in extenso en el anterior apartado:

The foregoing represents our impressions of the economic advice the Socialists leadership is receiving. The Party's actual economic program is in preparation and is due to be made public shortly. Although the program will obviously not be presented as starkly as we have outlined it, we expect that the basic policies will evolve as we have described them (68).

Puede resultar paradójico, pero parece fuera de toda duda razonable que millones de españoles, cuya opción fue dar su voto al Partido Socialista, estaban peor informados sobre la verdadera agenda económica del PSOE que la propia Administración Reagan. Esta circunstancia fue posible, únicamente, gracias a la excelente labor realizada por los consejeros de la Embajada estadounidense en Madrid. Una revisión del programa electoral del PSOE para los comicios de 1982, a pesar de sus calculadas ambigüedades, no resiste en el ámbito de la economía una comparación con las políticas posteriormente implementadas (69). Baste mencionar la incapacidad de combatir el paro a pesar de la promesa explícita de generar «más de 800.000 empleos netos durante los cuatro años de gestión gubernamental» (70).

norteamericanos David Rockefeller y Thomas Enders que su proyecto no entraría en colisión con los intereses de Estados Unidos en el marco de una reunión en Santo Domingo a la que también asistió Carlos Andrés Pérez, político venezolano con gran vinculación con el líder español y compañero en la Internacional socialista, MARQUINA BARRIO (1991): 35-36.

(67) «Spain: The New Party System. An Intelligence Assessment», Directorate of Intelligence, noviembre de 1982.

(68) NARA, RG 56, GRDT, OASIA, RRPIS 1976-1981, box 2: The Outlines of a Socialist Economic Policy in Spain, 14 de julio de 1982.

(69) Al lenguaje intencionadamente ambiguo del programa electoral del PSOE se refiere Ysàs (2011). Para un balance general de la política económica del primer mandato socialista RECIO y Roca (1998); Soto Carmona (2006); Fernández Navarrete y Matías (2006) y Marín (2008).

(70) «Por el cambio. Programa Electoral del Partido Socialista Obrero Español», Programa electoral del PSOE, 1982, p. 7. Todavía en 1984 Alfonso Guerra era reacio a asumir el fracaso del partido en esta empresa y aunque reconocía las dramáticas cifras de desempleo, decía estar convencido de haber creado ya por entonces 400.000 puestos de trabajo. GUERRA (1984): 148-149. 
A la vista de algunas de las revelaciones aquí recogidas es preciso abandonar ciertas ideas preconcebidas acerca de las relaciones entre la Administración Reagan y la llegada de los socialistas al poder. Evidentemente, entre ambos gobiernos se produjeron roces y existió una falta de sintonía que quedó perfectamente ejemplificada en algunos gestos un tanto estrambóticos que tuvieron lugar durante la visita del presidente norteamericano a Madrid en 1985 (71). Además, la política exterior del mandatario republicano contribuyó a que no se disipara el difuso sentimiento anti-norteamericano en una parte de la sociedad española que seguía identificando a Estados Unidos como una potencia imperialista que había contribuido a la supervivencia del régimen franquista. Sin embargo, en lo que a política económica se refiere, la distancia parece ser mucho menos pronunciada. La llegada de los socialistas al poder no conllevó una modificación sustancial de las relaciones en este ámbito y España siguió apareciendo, a los ojos estadounidenses, como un país serio y fiable con el que era posible hacer negocios. Esta continuidad manifiesta la existencia de más puntos en común de lo que un análisis superficial podría revelar. Tal circunstancia nos debe invitar a repensar hasta qué punto tuvieron éxito iniciativas educativas bilaterales -como puede ser el programa Fulbright- en la captación de los futuros líderes de la España reciente, así como cuál fue el verdadero papel que jugó la diplomacia pública norteamericana en la preparación del posfranquismo (72).

\section{REFERENCIAS}

\section{Archivos}

General Records of the Department of State (Record Group 59), Records of Henry A. Kissinger 1973-1977, National Archives at College Park (MD), EEUU.

General Records of the Department of the Treasury (Record Group 56), Office of the Assistant Secretary for International Affairs, Records Relating to Portugal, Italy and Spain 1976-1981, National Archives at College Park (MD), EEUU.

Claiborne Pell Papers, Special Collections, University of Rhode Island, Kingston (RI) EEUU.

(71) Powell (2011): 604-616. Calvo Sotelo sugiere que esta clase de gestos servían a los socialistas para lavar su mala conciencia por implementar una política económica de derechas: «la expropiación de RUMASA fue una guinda de izquierdas [...] como la espantá de Alfonso Guerra cuando vino Reagan o como esas otras guindas caras, sobre la política exterior que tan cuidadosamente colocaba en Nicaragua o en Angola Luis Yáñez Barnuevo», CALvo Sotelo (1990): 162.

(72) DELGADO (2009) y (2010). No fueron pocos los socialistas que tuvieron la oportunidad de ser becarios del programa Fulbright y, posteriormente, ocuparon cargos de alta responsabilidad. Los casos más conocidos son los de Javier Solana (1966) o Pasqual Maragall (1971) pero muchos otros simpatizantes del PSOE también se beneficiaron del mismo. Todos ellos reconocen la importancia de la experiencia y el cambio que en su concepción de la potencia americana provocó este intercambio. A su vez, otros políticos socialistas también pudieron disfrutar de estancias cortas en Estados Unidos en otros programas como los destinados a conocer a los jóvenes líderes. 


\section{BIBLIOGRAFÍA}

Álvaro, AdORACIÓN (2011): «Hízose el milagro. La inversión directa estadounidense y la empresa española (c. 1900-1975)», Investigaciones de Historia Económica, n. ${ }^{\circ}$, pp. 358-368.

ANDRADE BLANCO, JUAN A. (2007): «Del socialismo autogestionario a la OTAN: Notas sobre el cambio ideológico en el PSOE durante la transición a la democracia», Historia Actual Online, 14, pp. 97-106.

Andrew, Christopher (1995): For the President's Eyes Only, New York, Harper Collins.

AviLÉs, JuAN (2013): «El proyecto socialista: del marxismo a la modernización», en Álvaro Soto Carmona y Abdón Mateos (eds.): Historia de la época socialista. España: 1982-1996, Madrid, Sílex, pp. 21-42.

AzCÁRATE, MANUEl (1988): «La percepción española de Estados Unidos», Leviatán, revista de hechos e ideas, n. ${ }^{\circ}$ 33, pp. 5-18.

Bel, GeRmÁ y ANTÓN COSTAS (2001): La privatización y sus motivaciones en España: de instrumento a política», Revista de Historia Industrial, n. ${ }^{\circ} 19-20$, pp. 105-132.

Bell, Coral (1989): The Reagan Paradox. US Foreign Policy in the 1980s, New Brunswick (NJ), Rutgers University Press.

Bermeo, Nancy \& José García-Durán (1994): «Spain: Dual Transition Implemented by Two Parties», en Stephan Haggard \& Steven B. Webb (eds.): Voting for Reform: The Political Economy Adjustment in New Democracies, Oxford, Oxford University Press/World Bank.

BlázQuez, BelÉN (2006): La proyección de un líder político: Felipe González y Nicaragua 1978-1996, Sevilla, Centro de Estudios Andaluces.

Cabrera, Mercedes (2011): «Los pactos de la Moncloa: acuerdos políticos frente a la crisis», Historia y Política, n. ${ }^{\circ}$ 26, pp. 81-110.

- y Fernando Del Rey (2011): El poder de los empresarios. Política y economía en la España contemporánea (1875-2010), Barcelona, RBA.

Calvo Sotelo, Leopoldo (1990): Memoria viva de la transición, Barcelona, Plaza \& Janés/Cambio 16.

Chipman, John (ed.) (1988): «NATO’s Southern Allies: Internal and External Challenges», London, Routledge.

Chislett, William (2005): España y Estados Unidos. En busca del redescubrimiento mutuo, Barcelona, Ariel.

Comín, Francisco y DANiel, DíAz Fuentes (2004): La empresa pública en Europa: una perspectiva histórica, Madrid, Síntesis.

Delgado, Lorenzo (2009): Viento de poniente. El programa Fulbright en España, Madrid, LID.

(2010): «After Franco, What? La diplomacia pública de Estados Unidos y la preparación del posfranquismo», en ÓSCAR J. MARTín GARCía y MANUEL, ORTIZ HERAS (coords.): Claves internacionales en la transición española, Madrid, los libros de la catarata, pp. 99-127. 
Eaton, SAmuel (1981): The forces of Freedom in Spain 1974-1979, Stanford, Hoover Institution Press.

ESTEFANíA, JOAQUín (2007): La larga marcha, Barcelona, Península.

- (2013): «El segundo ajuste económico de la democracia», en Álvaro SoTO Carmona y Abdón Mateos (eds.): Historia de la época socialista. España: 19821996, Madrid, Sílex, pp. 127-146.

Feo, Julio (1993): Aquellos años, Barcelona, Ediciones B.

Fernández Navarrete, Donato y Gustavo Matías (2006): «Ajuste estructural de la economía española y negociaciones para entrar en las Comunidades Europeas durante el primer gobierno socialista (1982-1985)», Historia del presente, n. ${ }^{\circ} 8$, pp. 39-60.

Fernández Sola, Natividad \& Smith, Michael (2009): Perceptions and Policy in Transatlantic Relations. Prospective Visions from the US and Europe, New York, Routledge.

FERNÁNDEZ, DANIEL (2012): El enemigo yanqui. Las raíces conservadoras del antiamericanismo español, Zaragoza, Genueve Ediciones.

Fuentes Quintana, Enrique (1990): «De los Pactos de la Moncloa a la Constitución (julio 1977-diciembre 1978)», en José Luis García Delgado (dir.): Economía española de la transición y la democracia, Madrid, Centro de Investigaciones Sociológicas, pp. 23-34.

- (1999): «La economía española desde la transición al presente», en GonZALO ANES (ed.): Historia económica de España, siglos XIX y XX, Barcelona, Galaxia Gutenberg, pp. 717-750.

GÁlveZ, SERGIO (2013): Modernización socialista y reforma laboral, (1982-1992), tesis inédita, Madrid, Universidad Complutense de Madrid.

GÁMIR, LuIs (dir.) (2008): Política económica de España, Madrid, Alianza.

García Delgado, José Luis y JuAn CARlos, Jiménez (2003): «La economía», en José María Jover Zamora (dir.): Historia de España Menéndez Pidal, T. XLII. La transición a la democracia y el reinado de Juan Carlos I, Madrid, Espasa Calpe, pp. 377-429.

GiL, Federico \& Tulchin, JosePh S. (eds.) (1988): Spain's Entry into NATO. Conflicting political and Strategic Perspectives, Boulder (CO), Lynne Rienner Publishers, Inc.

GiLPIn, Robert (1987): The Political Economy of International Relations, Princeton, Princeton University Press.

Gomes, Bernardino y Moreira de Sá, Tiago (2008): Carlucci vs. Kissinger. Os EUA e a Revoluçao Portuguesa, Lisboa: Dom Quixote.

Guerra, Alfonso (1984): Felipe González. De Suresnes a la Moncloa, Madrid, Novatex.

Gunther, Richard; Diamandouros, Nikiforos P. y Puhle, Hans-Jürgen (1995): The Politics of Democratic Consolidation: Southern Europe in Comparative Perspective, Baltimore, Johns Hopkins University Press.

Gunther, Richard; Montero, José Ramón y Botella, JoAn (2004): Democracy in Modern Spain, New Haven, Yale University Press. 
HERnÁNDEZ, JuAn (2004): Economía política de la transición en España 1973-1980, Madrid, Editorial Complutense.

Hopkin, JonAthan (2000): El partido de la transición: ascenso y caída de la UCD, Madrid, Acento.

JARQUe, ARTURo (1998): Queremos esas bases. El acercamiento de Estados Unidos a la España de Franco, Madrid, Centro de Estudios Norteamericanos, Universidad de Alcalá.

JERVIS, ROBERT (1971): The Logic of Images in International Relations, Princeton, Princeton University Press.

(1976): Perception and Misperception in International Politics, Princeton, Princeton University Press.

y Robert Art (2014): International Policy: Enduring Concepts and Contemporary Issues, 12 edition, New York, Pearson.

LEMUS, ENCARNACIÓN (2011): Estados Unidos y la transición española, Madrid, Sílex.

Lemus, Encarnación; Rosas, Fernando y Varela, Raquel (coords.) (2010). El fin de las dictaduras ibérias (1974-1978), Sevilla/Lisboa, Centro Estudios Andaluces/ Ediçoes Pluma.

LinARES, ÁNGEL LUIS (2008): La política de seguridad de la transición española, 1976-1982, Madrid, Ministerio de Defensa.

López ZAPICO, MisAel A. (2013): Acciones y percepciones: la diplomacia, la economía política y la prensa escrita en las relaciones hispanonorteamericanas durante el tardofranquismo y los inicios del proceso democratizador, Huelva, Universidad de Huelva.

- (2011): «Anatomía de "un asunto interno". La actitud del gobierno estadounidense ante el 23-F», Ayer, n. ${ }^{\circ}$ 84, pp. 183-205.

MAluquer de Motes, Jordi (2008): El largo camino a Europa. Cincuenta años del Círculo de Economía, Barcelona, Círculo de Economía y Centro editor PDA.

Marín ArCe, José María (2008): «Los socialistas en el poder (1982-1996)», Historia y Política, n. ${ }^{\circ}$ 20, pp. 43-71.

MARQuina BARRIo, ANTONiO (1986): España en la política de seguridad occidental. 1939-1986, Madrid, Ejército.

- (1991): «Spanish Foreign and Defense Policy since Democratization», en KENNeTh Maxwell (ed.): Spanish Foreign and Defense Policy, Boulder (CO), Westview Press, pp. 19-62.

Mateos, Abdón (2013): «El PSOE de Felipe González. La transformación del partido», en Álvaro Soto Carmona y Abdón Mateos (eds.): Historia de la época socialista. España: 1982-1996, Madrid, Sílex, pp. 367-387.

Melanson, Richard A. (2005): American Foreign Policy since the Vietnam War, New York, M.E. Sharpe.

Molinero, CARme (2011): «El acceso a los archivos y la investigación histórica», Ayer, n. ${ }^{\circ}$ 81, pp. 285-297.

MuÑoz SÁnchez, ANTONIO (2012): El amigo alemán. El SPD y el PSOE de la dictadura a la democracia, Barcelona, RBA. 
NiÑo, ANTONiO y CARLOS SANZ (2012): «Los archivos, la intimidad de las personas y los secretos de Estado», Cuadernos de Historia Contemporánea, n. ${ }^{\circ} 34$, pp. 309342.

Niskanen, William. A. (1988): Reaganomics. An Insider's Account of the Policies and the People, Oxford, Oxford University Press.

ORTUÑo, PILAR (2005): Los socialistas europeos y la transición española, Madrid, Marcial Pons.

Papell, Antonio (1991): Conversaciones con Luis Yáñez, Barcelona, Plaza y Janes.

PARDO, Rosa (2003): «La política norteamericana», Ayer, n. ${ }^{\circ} 49$, pp. 13-53.

(2011): «La política exterior de los gobiernos de Felipe González: ¿un nuevo papel para España en el escenario internacional?», Ayer, n. ${ }^{\circ}$ 84, pp. 73-97.

Portero, Florentino (2003): «Las relaciones con Estados Unidos y la política de Seguridad», en José María Jover Zamora (dir.): Historia de España Menéndez Pidal, T. XLII. La transición a la democracia y el reinado de Juan Carlos I, Madrid, Espasa Calpe, pp. 785-794.

Powell, Charles (2007): «Estados Unidos y España, de la dictadura a la democracia: el papel de Henry A. Kissinger (1969-1977)», en Charles T. POWEll y JuAN CARLOS, JIMÉNEZ (eds.): Del autoritarismo a la democracia. Estudios de política exterior española, Madrid, Sílex, pp. 19-71.

- (2010): «El papel de Estados Unidos en la transición democrática española», en Óscar J. Martín García y Manuel Ortiz Heras (eds.): Claves internacionales en la transición española, Madrid, Los libros de la Catarata.

- (2011): El amigo americano, Barcelona, Galaxia Gutenberg.

PUIG, NURIA (2005): «La ayuda económica de EE.UU. y la americanización de los empresarios españoles», en LoRENZo Delgado, y M. ${ }^{a}$ Dolores, Elizalde (eds.): España y Estados Unidos en el siglo XX, Madrid, CSIC, pp. 181-205.

Reagan, Ronald (1990): An American Life, New York, Simon and Schuster.

(2009): The Reagan Diaries. Volume I, January 1981-October 1985, editado por Douglas Brinkley, New York, Harper Collins.

- (2011): The notes, editado por Douglas Brinkley, New York, Harper Collins.

Recio, AlBert y Jordi Roca (1998): «The Spanish Socialists in Power: Thirteen Years of Economic Policy», Oxford Review of Economic Policy, 14, pp. 139-158.

RODRIGO, FERNANDO (1995): «La inserción de España en la política de seguridad occidental», en Richard Gillespie, Fernando Rodrigo y Jonathan StORY (eds.): Las relaciones exteriores de la España democrática, Madrid, Alianza, pp. 77-103.

Rosendorf, Neal M. (2014). Franco Sells Spain to America, London, Palgrave Macmillan.

RUPÉREZ, JAVIER (1986): España en la OTAN: relato parcial, Barcelona, Plaza \& Janés.

SÁnchez Domínguez, M. a Ángeles \& M.a Ángeles Ortega Almón (2001): «The privatization process in Spain 1985-2001», en Teoria e Evidência Econômical Brazilian Journal of Theoretical and Applied Economics, vol. 9, n. ${ }^{\circ}$ 17, pp. 9-24.

SÁnCHEZ ReCIO, Glicerio (2008): «La percepción de los cambios como factor de la transformación cultural y social», en GLICERIO SÁNCHEZ RECIO (coord.): Eppure 
si muove. La percepción de los cambios en España (1959-1976), Madrid, Biblioteca Nueva, pp. 15-29.

SÁnCHEZ-Guón, ANTONio (1978): España en la OTAN, Madrid, Ediciones Defensa, 1978.

SEgURA, Julio (1998): «Intervención pública y política de bienestar: el papel del Estado», en José Luis García Delgado (dir.): España, economía, Madrid, Alianza, pp. 831-857.

SEREGNI, AlesSANDRO (2010): «Antiamericanismo y democracia», en ÓSCAR J. MARTín García y Manuel Ortiz Heras (coords.): Claves internacionales en la transición española, Madrid, los libros de la catarata, pp. 128-147.

Shultz, GeORge (1993): Turmoil and Triumph. My Years as Secretary of State, New York, Charles Scribners's Sons.

Soto Carmona, Álvaro (2006): «Felipe González más reformista que socialdemócrata: balance de una gestión», Historia del presente, n. ${ }^{\circ}$ 8, pp. 13-37.

Stabler, Wells (1987): «The View from the US Embassy», en Hans BinNENDiJK (ed.): Authoritarian Regimes in Transition, Washington D.C., Foreign Service Institute, US Department of State, pp. 192-197.

SUDRIÀ, CARLES (1987): «Un factor determinante: la energía», en JORDI NADAL et al. (comps.): La economía española en el siglo XX. Una perspectiva histórica, Barcelona, Ariel, pp. 313-363.

TASCóN, Julio (2005): «La red yanqui desde los años treinta hasta los años del milagro», en JUlio TASCóN (dir.): Redes de empresas en España. Una perspectiva teórica, histórica y global, Madrid, Lid, pp. 137-154.

TERmis Soto, Fernando (2005): Renunciando a todo. El régimen franquista y los Estados Unidos desde 1945 hasta 1963, Madrid, Biblioteca Nueva.

TRULlÉN, JoAn (1993): Fundamentos económicos de la transición política española: la política económica de los Acuerdos de la Moncloa, Madrid, Ministerio de Trabajo y Seguridad Social.

ViÑAS, ÁNGEL (2003): En las garras del águila, Barcelona, Crítica.

Voss, JAMES F. \& DORSEY, Ellen (1992): «Perceptions and International Relations: an Overview», en E. SINGER \& V. Hudson (eds.), Political Psychology and Foreign Policy. Boulder (CO): Westview Press.

WilentZ, SEAn (2009): The Age of Reagan: A history 1974-2008, New York, Harper Collins.

YSÀs, PERE (2011): «Cambio y continuidades: tres lustros de gobiernos socialistas», Ayer, n. ${ }^{\circ} 83$, pp. 23-49.

Zaldivar, CARlos (2003): «Miradas torcidas. Percepciones mutuas entre España y Estados Unidos», Working Paper, Real Instituto Elcano, accesible en www.realinstitutoelcano.org/documentos/60.asp 